(69)

\title{
Impacts of Meteorological Parameters on the Abundance of Malaria Vector in Batticaloa District, Sri Lanka
}

\author{
KannangaraD.N. ${ }^{1 *}$, RanathungeR.M.T.B. ${ }^{1}$, Abeyewickreme W. ${ }^{1}$, Hapugoda M.D. ${ }^{1}$, \\ SubasingheS.M.C.U.P. ${ }^{2}$ \\ ${ }^{1}$ Molecular Medicine Unit, Faculty of Medicine, University of Kelaniya \\ ${ }^{2}$ Department of Forestry \& Environmental Science, University of Sri Jayewardenepura, Sri Lanka \\ *niranjankannangara@gmail.com
}

\begin{abstract}
Malaria is one of the most widespread Vector-BorneDiseases (VBDs) in the world causing serious losses to the health and economy. Out of many factors, meteorological parameters effects on malaria distribution significantly. Conditions including higher temperature, lower relative humidity and higher wind velocity are found to be affecting on mosquito oviposition, propagation and survival. In Sri Lanka, Batticaloawas considered as one of the malaria endemicdistrictsduring recentpast. Control and effective management in these areas had been difficult due to the lack of reliable data, human resource and the deterioration of the infrastructure caused by years of armed conflict. This study was carried out to identify the relationships exist between different malaria vectors and meteorological parameters in the district of Batticaloa.Entomological surveillance was carried out in three potential malaria sensitive areas (Vakarai, Vakaneri and Mandur) with a radius of $20 \mathrm{~km}$ in the district of Batticaloa from April 2013 to July 2014. Adult mosquito samples were collected monthly by WHO standard method; Cattle Baited Net Collection (CBNT). Monthly meteorological parameters (T: mean temperature, RF: total rainfall, WS: wind speed and RH: Relative humidity) during the study period were also collected from the Department of Meteorology. Data were analyzed using SPSS version 15.0. Pearson's correlation coefficients (r) at 0.05 probability were used to investigate the relationships among meteorological variables of the current month along with lag time period of two months and vector densities. The most dominant mosquito species in the area was An. subpictuswith a relative abundance of $31.56 \%$ while An. nigerrimuswas the second highest (21.22\%). Significant relationships were only observed with RH and WS where, An. nigerrimus showed a negative moderate relationship ( $\mathrm{r}=0.372 ; \mathrm{p}=0.03$ ) while An. subpictus showed a positive relationship ( $\mathrm{r}=0.375 ; \mathrm{p}=0.029)$ with RH. In addition, An. subpictusalsoshowed a negative relationship with one month lagged time of WS. The findings of the study reveal that the relationships that exist between the vector and the meteorological parameters are complicated and unpredictable. Neither patterns nor consistencies were observed in these relationships. The adaptation of vector and the influence of other environmental and anthropological factors on the vector mosquito could be the reason for this behaviour.
\end{abstract}

Keywords:Malaria, Vector, Abundance, Relative humidity, Wind speed

Proceedings of the $22^{\text {nd }}$ International Forestry and Environment Symposium 2017 of the Department of Forestry and Environmental Science, University of Sri Jayewardenepura, Sri Lanka 